\section{Multiples Myelom: neue Therapieoptionen oder ASCT zur Konsolidierung?}

\author{
Standardtherapie für fitte Patienten mit neu diagnostiziertem multiplem \\ Myelom ist hochdosiertes Melphalan plus eine autologe Stammzelltransplan- \\ tation. Sind die neuen, weniger toxischen oralen Optionen dieser Strategie \\ überlegen?
}

$P$ atie atienten mit neu diagnostiziertem multiplem Myelom (MM), die jünger als 65 Jahre sind, haben nach autologer Stammzelltransplantation (ASCT) und Hochdosischemotherapie eine gute Prognose. Konkurrenz erhält dieses Vorgehen durch neue Substanzen wie Thalidomid, Lenalidomid und Bortezomib, die auch das Überleben von transplantationsfähigen Patienten verlängern - mit guter Verträglichkeit. In eine randomisierte Phase-III-Studie wurden 389 Patienten $(<65$ Jahre) mit neu diagnostiziertem MM eingeschlossen. Sie erhielten eine Standardinduktionstherapie mit Lenalidomid/Dexamethason sowie zur Stammzellmobilisation Cyclophosphamid und G-CSF („granulocyte-colony stimulating factor"). Randomisiert erfolgte bei geeigneten Patienten die Konsolidierung mit 6 Zyklen Cyclophosphamid/Dexamethason/Lenalidomid oder 2-mal Hochdosis-Melphalan plus ASCT. Anschließend wurden die Patienten auf eine Erhaltungstherapie mit Lenalidomid/Prednison oder Lenalidomid alleine randomisiert. Nach median 52,0 Monaten Follow-up war das progressionsfreie Überleben (PFS) nach der Konsolidie- rung mit Cyclophosphamid/Lenalidomid/Dexamethason signifikant kürzer als nach der ASCT (median 28,6 vs. 43,3 Monate; Hazard Ratio [HR] für die ersten 24 Monate 2,51; $\mathrm{p}<0,0001)$. Die Art der Erhaltungstherapie hatte keinen signifikanten Einfluss auf das PFS (37,5 Monate unter Lenalidomid/Prednison vs. 28,5 Monate unter Lenalidomid; $\mathrm{p}=0,34$ ).

Schwerwiegende Nebenwirkungen vom Grad 3/4 wurden unter MelphalanASCT häufiger gesehen als unter der Dreierkombination, wobei vor allem hämatologische, gastrointestinale und infektionsbedingte Ereignisse unter der ASCT deutlich häufiger auftraten (Tab. 1). Bei den Erhaltungstherapien gab es keine Unterschiede hinsichtlich der $\mathrm{Ne}$ benwirkungsinzidenz.

Fazit: Bei transplantationsfähigen Patienten mit neu diagnostiziertem MM ist die Konsolidierungstherapie mit Melphalan und ASCT im Vergleich zu Chemotherapie plus Lenalidomid nach wie vor die bessere Behandlungsoption im Hinblick auf das PFS. Die Verträglichkeit war aber unter Chemotherapie plus Lenalidomid besser. Bei der Erhaltungs-
Tab. 1: Nebenwirkungen unter den Konsolidierungstherapien (Auswahl)

\begin{tabular}{|l|l|l|l|l|l|l|}
\hline & \multicolumn{3}{l}{ CRD (n=129) } & \multicolumn{3}{l}{ MEL-ASCT (n=127) } \\
\hline Hämatologische NW & Grad 3 & Grad 4 & Grad 5 & Grad 3 & Grad 4 & Grad 5 \\
\hline Anämie & $24(7 \%)$ & $4(1 \%)$ & 0 & $16(12 \%)$ & $2(1 \%)$ & 0 \\
\hline $\begin{array}{l}\text { Neutropenie } \\
\text { Thrombozytopenie }\end{array}$ & $32(8 \%)$ & $8(2 \%)$ & 0 & $4(3 \%)$ & $98(77 \%)$ & 0 \\
\hline Nichthämatologische NW & $7(2 \%)$ & $1(<1 \%)$ & 0 & $15(12 \%)$ & $89(70 \%)$ & 0 \\
\hline Infektion & $24(6 \%)$ & $5(1 \%)$ & $2(1 \%)$ & $18(14 \%)$ & $6(5 \%)$ & 0 \\
\hline gastrointestinal & $16(4 \%)$ & $1(<1 \%)$ & 0 & $24(19 \%)$ & $1(1 \%)$ & 0 \\
\hline kardial & $9(2 \%)$ & 0 & $1(<1 \%)$ & $2(1 \%)$ & 0 & 0 \\
\hline
\end{tabular}

$N W=$ Nebenwirkungen; $C R D=$ Cyclophosphamid, Lenalidomid, Dexamethason; $M E L-A S C T=$ Hochdosis-Melphalan plus autologe Stammzelltransplantation therapie ergaben sich keine Unterschiede zwischen beiden Therapieoptionen.

Barbara Kreutzkamp

Gay F et al. Chemotherapy plus lenalidomide versus autologous transplantation, followed by lenalidomide plus prednisone versus lenalidomide maintenance, in patients with multiple myeloma: a randomised, multicentre, phase 3 trial. Lancet Oncol. 2015;16(16):1617-29.

\section{Kommentar von Prof. Einsele:}

Die von Francesca Gay und Kollegen publizierte Studie belegt erneut den Vorteil der Hochdosistherapie mit ASCT bei Patienten mit neu diagnostiziertem $M M$, die für eine solche Therapie infrage kommen. Diese Studienergebnisse sind im Einklang mit einer von Antonio Palumbo und Kollegen publizierten Arbeit, in der ebenfalls die Überlegenheit der Hochdosis-Therapie mit ASCT bei transplantationsfähigen Patienten gezeigt wurde [Palumbo A et al. N Engl J Med. 2014;371(10):895-905]. Hier wurde die Vergleichstherapie mit Melphalan/Lenalidomid plus Dexamethason durchgeführt. Unterstützt werden die Studienergebnisse auch durch Daten, die auf der letzten ASHJahrestagung von den französischen Kollegen vorgestellt wurden [Attal $\mathrm{M}$ et al. ASH.

\section{"Geeigneten Patienten sollte eine Hochdosistherapie mit ASCT empfohlen werden."}

2015;Abstr 391]. Auch in dieser Untersuchung war die Kombination aus Lenalido$\mathrm{mid} /$ Bortezomib plus Dexamethason einer Hochdosistherapie mit ASCT bei jüngeren Patienten unterlegen. Allerdings wurde bei diesen Patienten nur eine einmalige Hochdosistherapie plus ASCT appliziert.

Aufgrund dieser Daten ist es Konsens der nationalen, europäischen und internationalen Myelomforscher und Therapeuten, dass einem Patienten, der für eine ASCT geeignet ist (Alter bis 70 Jahre und keine wesentliche Komorbidität), eine Hochdosistherapie mit ASCT empfohlen werden sollte.

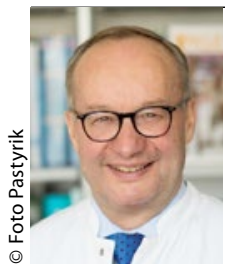

Prof. Dr. med. Hermann Einsele Med. Klinik und Poliklinik II am Universitätsklinikum Würzburg Einsele_H@ukw.de 